\title{
Three-Dimensional Digital Sonomicrometry: Comparison with Biplane Radiography
}

\author{
Pengcheng Shi ${ }^{\ddagger}$, Reza Mazhari ${ }^{\dagger}$, Donald Dione*, Jeffrey Omens ${ }^{\dagger}$, \\ Andrew McCulloch ${ }^{\dagger}$, and Albert Sinusas* \\ Departments of $\ddagger$ Electrical Engineering and *Medicine, Yale University, New Haven, CT 06520, USA \\ ${ }^{\dagger}$ Department of Bioengineering, University of California, San Diego, La Jolla, CA 92093 \\ email: Albert.Sinusas@yale.edu
}

\begin{abstract}
This paper describes a three-dimensional (3D) digital sonomicrometry approach for locating and tracking 3D objects. A commercial digital sonomicrometry system was employed to measure scalar distances between omnidirectional sonomicrometers. 3D coordinates were then derived using the statistical technique of multidimensional scaling (MDS). 3D digital sonomicrometry was directly compared with biplane radiography of the ultrasound crystals for estimation of $3 \mathrm{D}$ distances in static phantoms and in vivo using an experimental canine preparation. An excellent correlation $(\mathrm{r}=\mathbf{0 . 9 9 2})$ was seen when comparing intercrystal distances derived from biplane radiography and sonomicrometry $3 \mathrm{D}$ coordinate data in the gel phantom. A Bland-Altman analysis shows that the average difference in coordinate determined distance between these two different methodologies was only $0.63 \pm 0.46 \mathrm{~mm}$, over a range of inter-crystal distances of 3.14 to $17.28 \mathrm{~mm}$. In the in vivo canine preparation, the correlation between the sonomicrometry derived and biplane derived distances was also excellent $(r=0.992)$ with a slope of 1.05 and an intercept of 0.06. The Bland-Altman analysis shows that the average difference in coordinate determined distance between these two different methodologies was only $0.78 \pm 0.74 \mathrm{~mm}$, over a range of inter-crystal distances of 2.90 to $27.66 \mathrm{~mm}$. We have demonstrated the feasibility of accurately measuring scalar distances using 3D digital sonomicrometry. Digital sonomicrometry combines high spatial and temporal resolution with availability and portability to accurately measure distances in a closely packed array of implanted piezoelectric crystals.
\end{abstract}

\section{INTRODUCTION}

Digital sonomicrometry, with high temporal and spatial resolution, can be a useful tool in locating and tracking three-dimensional (3D) objects when used with the statistical technique of multidimensional scaling (MDS). The ability to locate an object's position in 3D space, and to observe the change in that position over time has numerous applications in the biological sciences. Cardiovascular applications include tracking a catheter as it moves through a blood vessel [12], investigating regional left ventricular (LV) deformation [3], and tracking the 3D geometry of the mitral valve [4]. Techniques involving 3D Magnetic Resonance Imaging (MRI), such as MR tagging and shape based boundary tracking may also be used for these purposes [10], [7]. However, these techniques employ beat averaging and long imaging times, which might cause them to miss crucial temporal changes in position. In addition, they have low spatial resolution in relation to sonomicrometry.

Biplane radiography using radiopaque markers is another method used to determine 3D wall motions and regional strains [9], [6], [2]. This technique, which uses perspective transformations to calculate the positions of implanted markers, offers relatively high spatial resolution $(<0.2 \mathrm{~mm})$ and temporal resolution $(120 \mathrm{~Hz})$. Digital sonomicrometry significantly improves spatial resolution $(0.024 \mathrm{~mm})$ and temporal resolution $(>125 \mathrm{~Hz})$. This may be particularly valuable for analyzing regional wall strains, since small errors in myocardial displacements can be amplified significantly in the computations [1].

Sonomicrometry also has the advantage of availability and portability that MRI and biplane radiography are lacking. However, it has yet to be validated in the in-vivo setting for the purposes of computing myocardial strain distributions.

In these experiments, 3D digital sonomicrometry is directly compared with biplane radiography of ultrasound crystals for estimation of 3D distances in static phantoms and in vivo using an experimental canine preparation.

\section{Methods}

\section{A. Digital Sonomicrometry}

A commercial digital sonomicrometry system (Sonometrics Corp., London, Canada) was employed to measure scalar distances between omni-directional sonomicrometers. Sonomicrometry utilizes the time-of-flight principle of ultrasound to calculate distances between a transmitter and a receiver. A high speed digital counter starts when a pulse of ultrasound is emitted by the transmitter, and is stopped when the first peak of the ultrasound wave is detected by the receiver. Using the known speed of ultrasound in tissue $(\sim 1540 \mathrm{~m} / \mathrm{s})$, the transit time is converted to a scalar distance. The distances between all possible pairs of crystals are recorded simultaneously for a given period of time, at a sampling frequency of greater than $100 \mathrm{~Hz}$.

Signal Processing. Due to several technical issues, the signals of recorded distances must be filtered to remove noise. This is accomplished through a two step process which involves first running the data through an automated filtering program, followed by a manual check. Occasionally, the system triggers on the second or subsequent ultrasound waves, rather than the leading edge. This leads to a "level shift" of the data trace, which is a brief increase in distance, corresponding roughly to a multiple of the ultrasound wavelength. Another type of error, a signal dropout, occurs when the system is triggered by external noise before the first peak reaches the receiver. The true distance data is a continuous and smooth trace of points. The continuity of the actual trace is examined by first and second order derivatives. The level-shift transition points are located, and the shifted segment is dropped down to its correct level. The dropout points are detected since they 
are below the true distance, and are interpolated into the trace with a tenth order least-squares polynomial. It is estimated that the automatic filter algorithm corrects over $90 \%$ of the data trace defects. The manual software then allows the user to visually examine the data traces, correct any remaining noise, and eliminate any crystal pair trace that is not correctable.

The filtered data is then passed to a $3 \mathrm{D}$ calculation program, which uses the MDS algorithm to convert the scalar distances to $3 \mathrm{D}$ coordinates. $\mathrm{MDS}$ is a statistical technique that spatially determines the similarities among a group of objects, such as inter-transceiver distances [8]. The MDS algorithm has two distinct phases. Simple geometric triangulation is used to obtain an initial estimate of the crystal coordinates. Next, a negative gradient iterative method calculates the final crystal positions by iteratively adjusting the $\mathrm{x}, \mathrm{y}, \mathrm{z}$ coordinates of each point in order to minimize the total error between the original measured distances and the same distances obtained from the reconstructed $3 \mathrm{D}$ data set. Simulated annealing technique is used to explore the entire solution space and avoid being trapped in any local minimum. In the derivation of crystal coordinates a value of $2 \mathrm{~mm}$ was added to each measured scalar crystal distance to correct for the center-to-center distance, assuming all crystals had a $2 \mathrm{~mm}$ diameter (1 mm radius, $\mathrm{x} 2$ crystals).

\section{B. Biplane Radiography}

Image Acquisition. Two x-ray projects are acquired in which all the markers (in these studies the crystals themselves were used as the biplane markers) are visible and distinct in both images. The images are recorded at 30 interlaced frames/sec. After the study is completed, a phantom is imaged at the same location as the experimental object to calibrate the images for magnification [6], [11].

Image Processing. After acquiring the images the centroid of each marker is located in each view using NIH Image, on a Macintosh (Quadra 950). Then, using stereo view projections, the $3 \mathrm{D}$ coordinates are calculated using previously established methods [6], [11]. The crystal coordinates were rotated into the same coordinate system.

\section{Experimental Evaluation}

Data presented here was collected utilizing a 3D triangular array of 12 ultrasound crystals embedded in a static phantom model, and an in vivo dynamic open chest canine preparation.

Static Gel Phantom. Three columns of four crystals $(n=12)$ were embedded in a cylinder-shaped polyacrilamide gel in a triangular array. Data were recorded simultaneously using both biplane radiography and sonomicrometry under two static conditions. Data were first recorded with the gel in the natural, undeformed state, and then after being deformed by placing a weight on the surface of the gel. Crystals of 2 and $0.75 \mathrm{~mm}$ diameter were used in an alternating pattern for each column, to facilitate identification of individual crystals on radiography.

Dynamic Canine Study. The same 12 crystal array employed in the phantom study, was implanted in anterior wall of the heart of an anesthetized open chest canine preparation. An additional 3 crystals were placed in the heart as a cardiac specific reference. These additional crys- tals were place at the apex and base of the LV and in the postero-lateral wall opposite the 12 crystal array. All inter-crystal distances among the 12 crystals in the anterior wall were compared. Although the 3 reference crystals were used to compute the $3 \mathrm{D}$ coordinates and cardiac local axes, distances to these reference crystals were not included in our statistical analysis. High fidelity micromanometers were placed in the LV and proximal aorta to define end-diastolic and end-systolic phases of the cardiac cycle. Crystal coordinates were computed for these two time points using both methodologies. Data were acquired under baseline conditions and in the presence of regional dysfunction produced by partial and complete occlusion of the left anterior descending coronary artery.

\section{Analysis}

Static Gel Phantom. For both biplane radiography and sonomicrometry approaches, the distances between each possible pair of crystals was calculated from their respective coordinates. A total of 66 crystal distances were available for analysis, for each static gel acquisition. All measured inter-crystal scalar distances were compared with inter-crystal distances computed from 3D crystal coordinates derived after application of MDS. Distances derived from biplane radiography were then compared with both the measured scalar crystal distances and distances computed from the 3D crystal coordinates. A linear regression was calculated and a Bland-Altman analysis was performed $[5]$.

Dynamic Canine Study. Distances derived from biplane radiography were compared with distances computed from the $3 \mathrm{D}$ crystal coordinates, as outlined above.

\section{RESULTS}

\section{A. Static Gel Phantom}

For the gel phantom there were a maximum of 132 intercrystal distances compared (66 distances $\mathrm{x} 2$ acquisitions). Only 57 of 66 potential crystal pairs had measured transit time signals which were analyzable. The correlation between measured scalar crystal distances and distances computed from derived crystal coordinates are shown in figure $1 \mathrm{a}$ and $\mathrm{b}$. These distances were highly correlated $(\mathrm{r}=0.956)$, although there was a mild $(0.52 \pm 1.03 \mathrm{~mm})$ systematic underestimation of measured distances relative to the distances calculated from crystal coordinates derived using MDS. This may reflect a difference in the applied offset correction for center-to-center distances. A standard $2 \mathrm{~mm}$ offset was applied in the MDS algorithm to correct for crystal thickness. In contrast, a variable adjustment was made in the measurement of inter-crystal transit time, based on the crystal sizes for each crystal pair.

The correlation between the measured crystal distances and biplane radiography derived distances was excellent $(\mathrm{r}=0.959)$, as illustrated in figure $2 \mathrm{a}$. There was no systematic error when comparing the measured distances and the distances derived from 3D x-ray coordinates (figure 2b). The best correlation $(\mathrm{r}=0.992)$ was seen when comparing inter-crystal distances derived from biplane radiography and sonomicrometry 3D coordinate data (see figure $3 \mathrm{a}$ ). The slope of this relationship was 0.95 with an intercept of only $0.10 \mathrm{~mm}$. The Bland-Altman analysis shows that the crystal coordinate data yields consistently longer distances 
than the biplane x-ray data (see figure $3 \mathrm{~b}$ ). The average difference in coordinate determined distance between these two different methodologies was only $0.63 \pm 0.46 \mathrm{~mm}$, over a range of inter-crystal distances of 3.14 to $17.28 \mathrm{~mm}$.

\section{B. Dynamic Canine Study}

A total of 528 inter-crystal distances (66 distances, x 2 phases of the cardiac cycle, $\mathrm{x} 4$ experimental conditions) were compared using both biplane radiography and sonomicrometry, in our in vivo canine preparation. The correlation between the sonomicrometry derived and biplane derived distances was excellent $(\mathrm{r}=0.992)$ with a slope of 0.93 and an intercept of 0.16 (figure $4 \mathrm{a}$ ). The BlandAltman analysis shows that the crystal derived coordinate data gave on average a longer distance than the biplane radiography. The average difference in coordinate determined distance between these two different methodologies was only $0.78 \pm 0.74 \mathrm{~mm}$, over a range of inter-crystal distances of 2.90 to $27.66 \mathrm{~mm}$ (see figure $4 \mathrm{~b}$ ).

\section{Discussion}

We have demonstrated the feasibility of accurately measuring scalar distances using 3D digital sonomicrometry. We have also shown that the multidimensional scaling algorithm is effective in converting the measured scalar distances into a system of 3D coordinates from which we can derive more accurate non-scalar distances. We have effectively applied this $3 \mathrm{D}$ sonomicrometry approach to in vivo tissue in a dynamic cardiac preparation, providing a unique method for defining complex regional deformations in the heart. 3D displacements derived from the measured distances could be used to compute non-homogeneous strains in the beating heart.

Bland-Altman analysis was applied in the comparison of the proposed 3D digital sonomicrometry approach with the more established biplane radiographic approach to determine whether they agree sufficiently for the new approach to replace the accepted standard for the measurement of regional deformations. This is very different from a more standard calibration, in which known quantities are measured by a new technique, and the measured values are compared to the true values. The Bland-Altman analysis was used to assess the degree of agreement between the two methods since neither provides an unequivocally correct measurement. This is important since the sonomicrometry approach has potentially a better spatial and temporal resolution. This analysis demonstrated a consistent overestimation of the crystal distances compared with the distances derived from biplane radiography. In our in vivo canine preparation, this overestimation was small $(<0.78 \mathrm{~mm})$ and independent of measured distance, suggesting that the $3 \mathrm{D}$ digital sonomicrometry could be used to compute regional strains comparable to biplane radiography.

The reason for the small discrepancy is computed distances using both methodologies remains uncertain, although several potential explanations exist. Differences could be attributed to independent know errors of each technology. The measurement of distance between two piezoelectric crystals has four possible sources of error. One is the accuracy of the time measurement, the second involves adding or subtracting an offset to the calculating distance, the third involves variations in the speed of sound

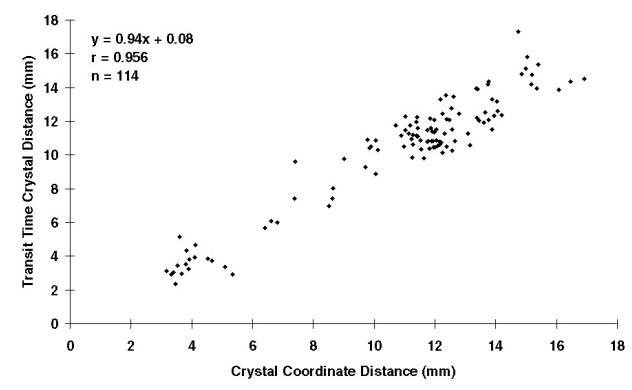

(a)

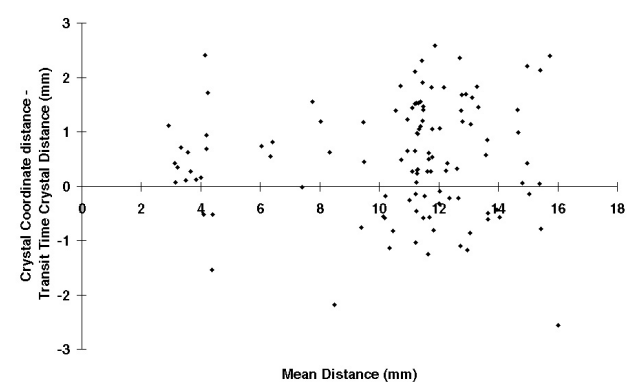

(b)

Fig. 1. (a): Transit time crystal distances are plotted versus coordinate crystal distances from the gel phantom. (b): A BlandAltman graph with the average distance of the two measurements plotted on the X-axis and the difference of the two measurements on the Y-axis.

in the medium or tissue, and the fourth is the accuracy of the reception of the signal by the receiver. Most likely the differences observed in the distances can be attributed to application of an incorrect offset for estimation of the center-to-center crystal distance. We applied a uniform correction of $2 \mathrm{~mm}$ for determination of crystal coordinates, however, employed both $2 \mathrm{~mm}$ and $0.75 \mathrm{~mm}$ crystals in our experiments. Further analysis using a variable correction could resolve this issue. In the future, the accuracy of the sonomicrometry approach could be improved by calibration of each crystal using a gig which fixes the crystals at a know distance in the material or tissue under evaluation.

\section{Conclusion}

3D digital sonomicrometry shows a high degree of agreement with the accepted technique of biplane radiography for measurement of distances in both static phantoms and contracting myocardial tissue. Digital sonomicrometry combines high spatial and temporal resolution with availability and portability to accurately measure distances in a closely packed array of implanted piezoelectric crystals. When combined with multidimensional scaling, sonomicrometry can effectively determine an object's position in three dimensional space, and is a technique which may have numerous applications in medical science.

\section{REFERENCES}

[1] McCulloch AD and Omens JH. Non-homogeneous analysis of three-dimensional transmural finite deformation in canine ventricular myocardium. J. Biomechanics, 24:539-548, 1991.

[2] Hashima AR, Young AA, McCulloch AD, and Waldman LK. Non-homogeneous analysis of epicardial strain distributions dur- 


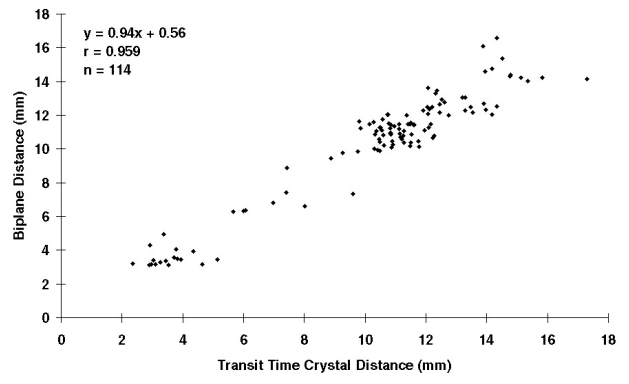

(a)

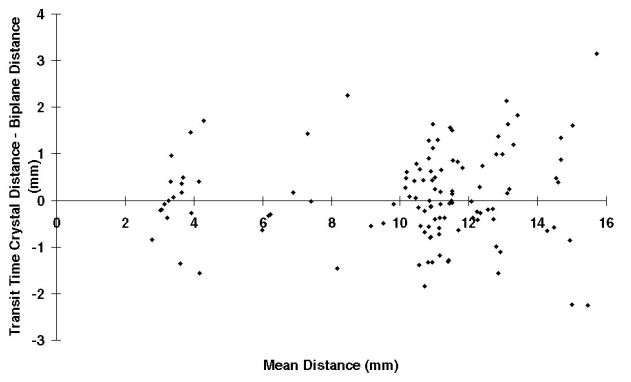

(b)

Fig. 2. (a): Transit time crystal distances are plotted versus Biplane distances from the gel phantom. (b): A Bland-Altman graph, with the average distance of the two measurements plotted on the $\mathrm{X}$-axis and the difference of the two measurements on the Y-axis.

ing acute myocardial ischemia in the dog. Journal of Biomechanics, 26:19-35, 1993

[3] Dione DP, Shi P, Smith W, DeMan P, Soares J, Duncan J, and Sinusas A. Three-dimensional regional left ventricular deformation from digital sonomicrometry. In Proceedings of the 19th Annual International Conference of the IEEE Engineering in Medicine and Biology Society, pages 848-851, 1997.

[4] Gorman JH, Gupta KB, Streicher JT, Gorman RC, Jackson BM, Ratcliffe MB, Bogen DK, and Edmunds LH Jr. Dynamic threedimensional imaging of the mitral valve and left ventricle by rapid sonomicrometry array localization. J Thorac Cardiovasc Surg, 112:712-726, 1996.

[5] Bland JM and Altman DG. Statistical methods for assessing agreement between two methods of clinical measurement. Lancet, pages 307-310, 1986.

[6] May-Newman K, Omens JH, Pavelec RS, and McCulloch AD. Three-dimensional transmural mechanical interaction between the coronary vasculature and passive myocardium in the dog. Circulation Research, 74:1166-1178, 1994.

[7] Axel L and Dougherty L. MR imaging of motion with spatial modulation of magnetization. Radiology, 171:841-845, 1989.

[8] Ratcliffe MB, Gupta KB, Streicher JT, Savage EB, Bogen DK, and Edmunds LH. Use of sonomicrometry and multidimensional scaling to determine the three dimensional coordinates of multiple cardiac locations: feasibility and initial implementation. IEEE Trans Biomed Eng, 42:587-598, 1995.

[9] Ingels N, Daughters G, Stinson E, and Alderman E. Measurement of midwall myocardial dynamics in intact man by radiography of surgically implanted markers. Circulation, 52:859-867, 1975.

[10] Shi PC, Sinusas AJ, Constable RT, Ritman E, and Duncan JS. Point-tracked quantitative analysis of left ventricular motion from $3 \mathrm{~d}$ image sequences. IEEE Transactions on Medical Imaging (in press).

[11] MacKay SA, Potel MJ, and Rubin JM. Graphics methods for tracking three-dimensional heart wall motion. Computers Biomedical Research, 15:455-473, 1982.

[12] Meyer SA and Wolf PD. Application of sonomicrometry and multidimensional scaling to cardiac catheter tracking. IEEE Transactions on Biomedical Engineering, 44:1061-1067, 1997.

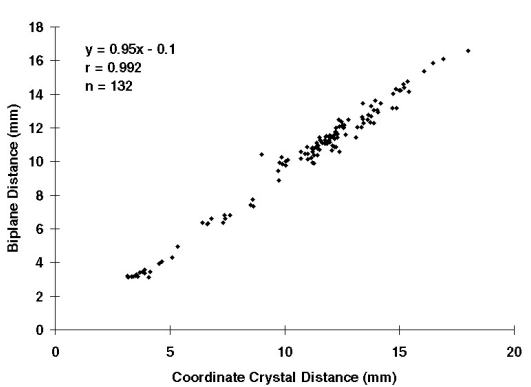

(a)

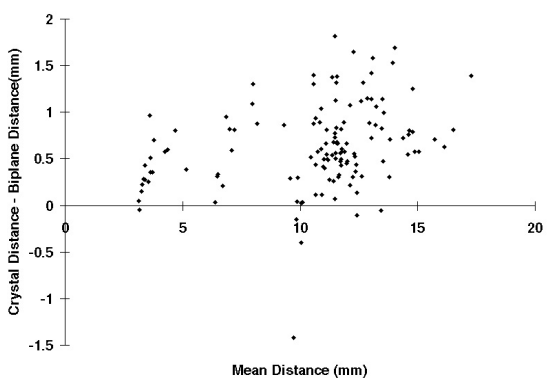

(b)

Fig. 3. (a): Crystal coordinate distances are plotted versus Biplane distances from the gel phantom. (b): A Bland-Altman graph, with the average distance of the two measurements plotted on the $\mathrm{X}$-axis and the difference of the two measurements on the $\mathrm{Y}$-axis.

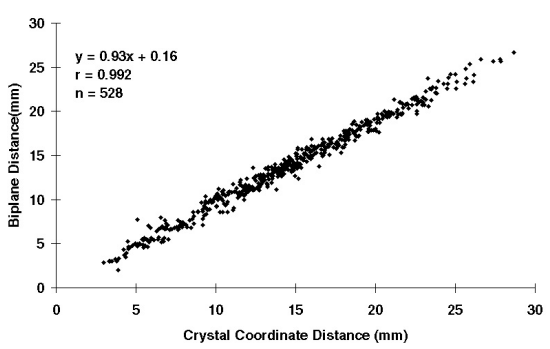

(a)

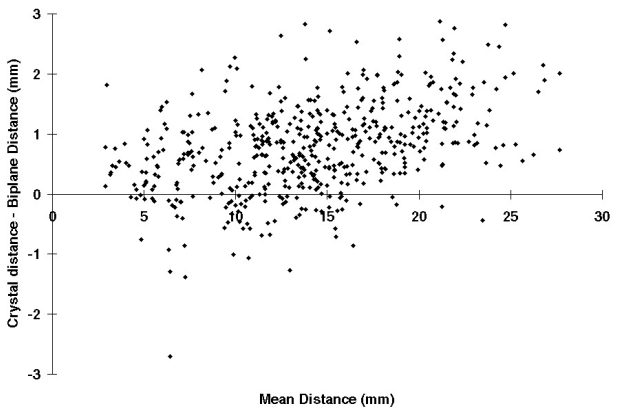

(b)

Fig. 4. (a): Crystal coordinate distances are plotted versus Biplane distances from the in vivo dog. (b): A Bland-Altman graph, with the average distance of the two measurements plotted on the $\mathrm{X}$-axis and the difference of the two measurements on the Y-axis. 